prednisolone; thiopurines; biologics). Rates are expressed as counts per 1,000 clinical events.

Results Comparing Apr-Jun 2020 (post-COVID) to Apr-Jun 2019 (pre-COVID): Total clinical event fell (9975 to 8208; $18 \%$ ), with a sharp drop in F2F OPD (3436 to 1203; -65\%) accompanied by a compensatory rise in non-F2F (1777 to 3161 ; +78\%). Rate of new diagnoses fell (49 to 13 per 1,000 events; -74\%). Prescription rates reduced sharply for thiopurines (26 to $5 ;-81 \%$ ), with lesser reductions for biologics $(89$ to $55 ;-38 \%)$ and oral prednisolone (25 vs $20 ;-20 \%)$ but with a rise for non-prednisolone steroids (5 vs 8 ; $+60 \%$ ). No change in relative proportion of different biologic classes. Conclusions Records of patient contacts were reduced in the immediate post-COVID period with a rapid shift from F2F to non-F2F. The drop in new patient records may reflect delayed pathways. Prescribing trends suggest a selective reduction in thiopurine and some shift from systemic to more topically-acting steroids. Longer term trends will be presented.

Acknowledgements Presented on behalf of sites collaborating in the UK IBD Registry.

\section{PMO-38 CONCOMITANT 5-AMINOSALICYLATE USE IN INFLAMMATORY BOWEL DISEASE PATIENTS ON BIOLOGIC THERAPY: A PRELIMINARY COST ANALYSIS}

Khai Leow*, Ramanan Gukathasan, Madeleine Bates, Sarah Alfred, Alison Danahay, Vivek Chhaya. University Hospitals Sussex NHS Foundation Trust, Worthing, UK

\subsection{6/gutjnl-2021-BSG.177}

Introduction Despite the rapidly evolving therapeutic armamentarium for inflammatory bowel disease (IBD), 5-aminosalicylates (5-ASAs) are still widely utilised as induction therapy and in maintenance of remission. Emerging data advocating the withdrawal of 5-ASAs in patients receiving biologic therapy ${ }^{1-4}$ presented an opportunity for us to evaluate local practice in honing a value-based healthcare model.

Methods IBD patients receiving biologic therapy from December 2019 to December 2020 were identified via the Blueteq High Cost Drugs system. A retrospective analysis was then carried out, aided by a pre-designed proforma. Data on patient demographics, IBD subtype, type of biologic therapy and concomitant 5-ASA use including the brand name, daily dose and subsequent annual cost were collected.

Results 382 patients were on biologic therapy over the 12month period, consisting of 204 males (53.4\%) and 178 females (46.6\%). A significant proportion were treated for Crohn's disease $(n=245,64.1 \%)$, followed by ulcerative colitis $(n=131,34.3 \%)$ and IBD-unclassified $(n=6,1.6 \%)$. Adalimumab therapy was encountered in 151 cases (39.5\%), Vedolizu$\mathrm{mab}$ in 93 cases (24.3\%), Infliximab in 79 cases $(20.7 \%)$, Ustekinumab in 46 cases (12\%), Tofacitinib in 9 cases $(2.4 \%)$ and Golimumab in 4 cases (1.1\%).

90 patients on biologic therapy were on concomitant 5ASAs (23.6\%). The vast majority suffered from ulcerative colitis $(n=65,72.2 \%)$, followed by Crohn's disease $(n=23$, $25.6 \%)$ and IBD-unclassified $(n=2,2.2 \%)$. The total annual cost of 5-ASA therapy in this cohort was estimated at $£ 73000$.

Conclusions In a pressing economic landscape, discontinuation of 5-ASAs on escalation to biologic therapy may yield significant cost savings which could be reinvested into other aspects of patient care in IBD. Rationalisation of treatment will also address the issue of non-adherence due to polypharmacy and consequently lead to improved clinical outcomes.

\section{REFERENCES}

1. Singh S, Proudfoot JA, Dulai PS, et al. No benefit of concomitant 5-aminosalicylates in patients with ulcerative colitis escalated to biologic therapy: pooled analysis of individual participant data from clinical trials. Am J Gastroenterol. 2018;113:1197-1205

2. Ma C, Kotze PG, Almutairdi $A$, Jairath V, Panaccione $R$, et al. Concomitant use of aminosalicylates is not associated with improved outcomes in patients with ulcerative colitis escalated to vedolizumab. Clin Gastroenterol Hepatol. 2019:17:2374-2376

3. Loftus E, Peyrin-Biroulet L, Vermeire $\mathrm{S}$, et al. P055 vedolizumab treatment persistence up to 5 years. Am J Gastroenterol. 2019;114:S14-S15.

4. Ungaro $R$, Limketkai $B$, Jensen $C$, et al. Stopping mesalamine therapy in patients with Crohn's disease starting biologic therapy does not increase risk of adverse outcomes. Clin Gastroenterol Hepatol. 2019;18:1152-1160.

\section{PM0-39 FACTORS INFLUENCING DELAYS IN BIOLOGIC INITIATION IN INFLAMMATORY BOWEL DISEASE}

${ }^{1}$ Eleanor Liu*, ${ }^{2} \mathrm{R}$ Jatale, ${ }^{3} \mathrm{D}$ DeSilva, ${ }^{3} \mathrm{~S}$ Hussain, ${ }^{1} \mathrm{Y}$ Danso, ${ }^{1} \mathrm{~J}$ Sabine, ${ }^{1} \mathrm{~J}$ Taylor, ${ }^{1} \mathrm{H}$ Sattar, ${ }^{3} \mathrm{PJ}$ Smith, ${ }^{3} \mathrm{~S}$ Subramanian, ${ }^{1} \mathrm{JK}$ Limdi. ${ }^{1}$ Pennine Acute Hospitals NHS Trust, Manchester, UK; ${ }^{2}$ Metropolis Healthcare, Mumbai, India; ${ }^{2}$ Liverpool University Hospitals NHS Foundation Trust, Liverpool, UK

\subsection{6/gutjnl-2021-BSG.178}

Introduction Biologics have transformed the treatment landscape in inflammatory bowel disease (IBD). Biologic initiation a multi-step process often associated with delays. We aimed to describe factors affecting 'decision to needle' (D2N) time in a real-world IBD cohort, identifying barriers to efficient delivery of biologic therapy.

Methods This was a retrospective review of IBD patients commenced on biologic therapy between December 2018 and December 2019 at 2 large IBD centres. Inpatient biologic initiations were excluded. We recorded patient demographics, Harvey Bradshaw Index (HBI)/partial Mayo score (pMayo)/ simple colitis activity index (SCCAI) and biochemical markers (faecal calprotectin [FC], C-reactive protein [CRP]). Dates were gathered on decision to treat, screening, blueteq approval and first dose. We used 21 days from decision to treat as a cut-off for determining D2N delay. Logistic regression examined associations between variables and D2N. Multivariate analysis was performed including variables with $\mathrm{p}<0.1$ from univariate analysis.

Results We included 204 patients (mean age 45 years; range 18-82 (Table 1)) and median disease duration of 6 years. Mean Charlson comorbidity index was 1 (range 0 -7). Adalimumab was initiated in 93 patients (46\%), ustekinumab:44 (22\%), vedolizumab:34 (17\%), infliximab:32 (16\%) and golimumab(1). Median D2N time was 44.5 days overall, for intravenous (IV) drugs $(n=110) 39.5$ days (range 1-264), and subcutaneous (SC) drugs $(\mathrm{n}=94) 50$ days (range 8-362). There were no significant associations in $\mathrm{D} 2 \mathrm{~N}$ time with age $(p=0.24), \quad$ gender $\quad(p=0.777)$, comorbidities $(p=0.908)$, employment $(p=0.478)$ or IBD diagnosis $(p=0.739)$. SC initiation was significantly associated with $\mathrm{D} 2 \mathrm{~N}$ delay in comparison to IV initiation $(p=0.004)$; OR 3.951 (CI 1.359 to $11.489 \mathrm{p}=0.016)$. Screening duration was significantly associated with $\mathrm{D} 2 \mathrm{~N}$ time on univariate $(\mathrm{p}=0.007)$, but not on multivariate analysis. Odds of D2N delay were lower with more severe baseline disease activity (OR 0.252 , CI 0.080 to $0.790 \mathrm{p}=0.018)$ and baseline $\mathrm{CRP}>5(\mathrm{p}=0.008)$. 


\begin{tabular}{ll} 
Abstract PMO-39 Table 1 & \\
\hline Baseline characteristics & N (\%) \\
\hline Female & $105(54)$ \\
IBD diagnosis & \\
Crohn's Disease & $127(62)$ \\
Ulcerative Colitis & $74(36)$ \\
IBD-unclassified & $3(1)$ \\
Prior biologic & $68(33)$ \\
MDT prior to decision & $57(28)$ \\
Corticosteroid at decision & $63(32)$ \\
Corticosteroid while awaiting initiation & $87(43)$ \\
\hline
\end{tabular}

Conclusions We noted that many patients experience a delay in biologic initiation after a clinical decision to treat. Delay was more likely with a SC route, likely attributable to drug delivery and healthcare at home availability. With the everincreasing demand for biologic therapy in IBD, streamlining the initiation process is imperative to minimise delay in starting effective therapy.

\section{PMO-40 COMPARISON OF THE PARTIAL MAYO AND PUCAI SEVERITY SCORES IN ULCERATIVE COLITIS}

\footnotetext{
1,2Stephanie Shields*, ${ }^{2}$ Konstantinos Gerasimidis, ${ }^{1,2}$ Jonathan Macdonald, ${ }^{3}$ Allan Dunlop, ${ }^{3}$ Peter Galloway, ${ }^{2}$ Vaios Svolos, ${ }^{2} \mathrm{Mr}$ Konstantinos Gkikas, ${ }^{4}$ Richard Hansen, ${ }^{1,2} \mathrm{~J}$ ohn Paul Seenan. 'Department of Gastroenterology, Queen Elizabeth University Hospital, Glasgow, UK; ${ }^{2}$ School of Medicine, Dentistry and Nursing, University of Glasgow, Glasgow, UK; ${ }^{3}$ Department of Biochemistry, Queen Elizabeth University Hospital, Glasgow, UK; ${ }^{4}$ Royal Hospital for Children, Glasgow, UK
}

\subsection{6/gutjinl-2021-BSG.179}

Introduction To assess disease severity in Ulcerative Colitis, the Partial Mayo (P-Mayo) score is used in adults and the Paediatric Ulcerative Colitis Activity Index (PUCAI) score is used for paediatric patients. The P-Mayo score uses an assessment of bowel frequency, rectal bleeding and a physician's global assessment to provide a score. The PUCAI score uses bowel frequency and rectal bleeding, but also incorporates abdominal pain, nocturnal rising, stool consistency and activity levels, arguably providing a more detailed overview. To our knowledge, the scores have never been directly compared. Our aim was to investigate how the scoring systems correlated with each other, and with biomarkers of disease activity.

Methods P-Mayo and PUCAI score, stool and serum samples were collected prospectively from 20 patients at set timepoints between day 0 and 42 after initiation of infliximab therapy. Albumin results were extracted from electronic patient records. Faecal Calprotectin (FC) was measured using a commercially available ELISA assay (CALP0170, Calpro AS). P-Mayo and PUCAI scores were classified as remission, mild, moderate or severe disease activity. R Studio was used to perform Pearson's correlation and agreement was assessed with Cohen's $\kappa$ tests.

Results 134 P-Mayo and 133 PUCAI scores, 131 albumin and 117 FC samples were included from 20 Patients (10 requiring infliximab as rescue therapy having met Travis criteria as inpatients), aged 14-70 (median 38) years. A strong correlation was seen between P-Mayo and PUCAI $(r=0.85, \mathrm{P}<0.0001$, CI 95\%: 0.80, 0.89). Better correlations were seen between PUCAI scores with FC and albumin, when compared to the P-Mayo Score (Table 1). Interclass agreement was moderate

\begin{tabular}{lllll}
\multicolumn{5}{l}{ Abstract PMO-40 Table 1} \\
\hline $\begin{array}{l}\text { Dataset } \\
1\end{array}$ & Dataset & Correlation & $95 \% \mathrm{Cl}$ & P-Value \\
\hline P-Mayo & FC & 0.46 & 0.30, & $<0.0001$ \\
& & & 0.59 & \\
PUCAI & FC & 0.57 & 0.43, & $<0.0001$ \\
& & & 0.68 & \\
P-Mayo & Albumin & -0.35 & $-0.50,-$ & $<0.0001$ \\
& & & 0.19 & \\
PUCAI & Albumin & -0.49 & $-0.62,-$ & $<0.0001$ \\
& & & 0.35 & \\
Mayo & PUCAI & 0.85 & 0.80, & $<0.0001$ \\
& & & 0.89 & \\
\hline
\end{tabular}

between P-Mayo and PUCAI scores $(\mathrm{k}=0.44)$; this improved slightly when mild and moderate disease activity was combined $(\kappa=0.51)$.

Conclusions In this small cohort of mostly adults, P-Mayo and PUCAI correlated strongly with moderate interclass agreement. PUCAI presented stronger correlations than P-Mayo score with blood and colonic disease biomarkers. External validation and evaluation against endoscopy is warranted.

\section{PM0-41 INCORPORATING HLADQA1*05 IN PRE-BIOLOGIC SCREENING IN IBD PATIENTS INITIATING BIOLOGIC THERAPIES}

Haidee Aleman Gonzalez*, Sankaranarayanan Ramachandran, Emma Whitehead, Alison Pattinson, Katie Stamp, Jack Turnbull, Sally Myers, Alison Talbot, Shaji Sebastian. Hull University Teaching Hospitals NHS Trust, Hull, UK

\subsection{6/gutjnl-2021-BSG.180}

Introduction The PANTS study reported high risk of immunogenicity and loss of response in anti Tumor Necrosis Factor (anti-TNFs) treated Crohn's disease (CD) patients carrying HLADQA1*05 allele. The proposed biomarker stratified trial to evaluate the usefulness of HLA testing prior to initiation of anti-TNFs is not yet available.

Aim To evaluate the use of HLADQA1*05 as part of pre-biologic screening in IBD patients initiating biologics on MDT decision on drug choice and disease outcomes

Methods We prospectively included all IBD patients who had HLADQA1*05 tested prior to initiation of biologics over a period of 12 months. Patients with definitive indication for one class of drug or drug strategy (perianal fistula, acute severe colitis, contraindications to infliximab, co-existent EIMs) were excluded. Primary outcome was treatment persistence at 6 and 12 months. Secondary outcomes were steroid free remission at 6 and 12 months, use concomitant immunosuppression and proportion needing dose escalation.

Results Seventy-six patients were included in analysis $(\mathrm{UC}=$ 32, $\mathrm{CD}=43$, IBD-U $=1$ ). HLADQA $1 * 05$ was positive in $46.7 \%$ of patients. The therapy class choice was as recorded in figure 1. Concomitant immunosuppression was used in $44 \%$ of the whole cohort and in $100 \%$ of HLADQA $1 * 05$ positive patients started on anti-TNF agents. Primary nonresponse was recorded in 8 patients and secondary loss of response in 3 patients. Among patients started on anti-TNFs, anti-drug antibodies were detected in 10 (15.6\%) patients 